\title{
The type material of Australian Cleridae (Coleoptera: Cleroidea) described by Eugen Hintz
}

With 8 figures

JUSTIN S. BARTLETT ${ }^{1}$

${ }^{1}$ Queensland State Government - Department of Agriculture and Fisheries, GPO Box 267, Brisbane, Queensland 4001, Australia. - justin.bartlett@daf.qld.gov.au

Published on 2015-12-21

\section{Summary}

The status of type specimens of Australian Cleridae described by EUGEN HINTz is assessed in accordance with the International Code of Zoological Nomenclature. Specimens are recognised as holotypes or syntypes with lectotypes being designated from syntype series. High resolution photographs of type material from the Senckenberg Deutsches Entomologisches Institut, Müncheberg are presented.

\section{Key words}

Phlogistus, type specimen status, lectotype designation

\section{Zusammenfassung}

Der Status der Typusexemplare der von Eugen Hintz beschriebenen Australischen Cleriden wird nach den Regeln des Internationalen Codes der Zoologischen Nomenklatur bewertet. Die Exemplare werden als Holotypen oder Syntypen festgelegt. Aus Serien von Typen wird ein Lectotypus ausgewählt. Hochaufgelöste Bilder des Typenmaterials aus dem Senckenberg Deutschen Entomologischen Institut in Müncheberg sind abgedruckt.

\section{Introduction}

Eugen Hintz (b. 1868, Danzig; d. 1932, Berlin) was a German engineer and non-professional entomologist who collected insects in Africa (STRAND 1917, Groll 2013) and published taxonomic papers dealing with Coleoptera; mainly Cleridae (Hintz 1902a, 1902b, 1905, 1907, 1908) and Cerambycidae (e.g., Hintz 1911, 1912, 1913, 1919). The majority of Hintz' clerid publications deal with African species, however, one paper deals with the endemic Australian genus Phlogistus Gorham. Hintz (1908) described nine species of Phlogistus and erected Phlogistomorpha Hintz to which he assigned Phlogistus blackburni SCHENKLING as its type. All but one of Hintz' Phlogistus species remain valid with BARTLETT (2009) proposing Phlogistus bicolor BARTLETt as a replacement name for Phlogistus blackburni Hintz (a junior homonym of Phlogistus blackburni SCHENkLING).

Type material of Hintz's Australian taxa was documented by the author during visits to three European museums (Museum national d'Histoire naturelle, Paris, France, Senckenberg Deutsches Entomologisches Institut, Müncheberg, Germany, and Museum für Naturkunde der Humboldt-Universität, Berlin, Germany); with the material from Senckenberg Deutsches Entomologisches 
Institut being borrowed for further study and photographic documentation. In this paper the status of type specimens of Hintz' Australian clerid taxa is assessed, lectotypes are designated where necessary, and photographs of type material from Senckenberg Deutsches Entomologisches Institut are presented.

\section{Materials and methods}

The fourth edition of the International Code of Zoological Nomenclature (ICZN 1999) was consulted to determine the status of Hintz' type material. Lectotypes are designated in accordance with Article 74.1, which states that lectotypes must be designated from syntypes, and Article 73.2, which states that all specimens of the type series are automatically syntypes if neither a holotype nor a lectotype has been fixed previously. Article 73.1.2 was referred to when recognising a type specimen as a holotype 'by monotypy'.

Specimen images were constructed with the aid of Helicon Focus montage software from photographs taken through a Nikon SMZ1500 stereo dissecting microscope with a Prior Proscan II stepping-motor and a Nikon DS U2/DS-Ri1 digital image capture system.

Abbreviations of museums are: MNHN, Museum national d'Histoire naturelle, Paris, France; SDEI, Senckenberg Deutsches Entomologisches Institut, Müncheberg, Germany; ZMHB, Museum für Naturkunde der Humboldt-Universität, Berlin, Germany.

The symbol ' $/$ ' signifies a break between individual labels.

\section{Assessment of type material}

\section{Phlogistus bicolor BARTLETT, 2009}

(Fig. 1)

Phlogistus blackburni Hintz, 1908: 714. Type locality: Australia. Unavailable - occupied by Phlogistus blackburni ScheNkLING, 1906 [current combination Phlogistomorpha blackburni (SCHENKLING, 1906)].

Phlogistus bicolor BARTLETT, 2009: 97. Replacement name for Phlogistus blackburni Hintz, 1908.

Lectotype (here designated): Peak Downs Mus. Godeffroy / Coll. S. Schenkling / Blackburni Determ. E. Hintz / Hintz det. / SYNTYPUS / Phlogistus bicolor Bartlett, 2009 nom. nov. Det. JS Bartlett Aug. 2011 (SDEI).

Paralectotype (here designated): Austral. / Type / Blackburni Hintz, Determ. E. Hintz. / ex Musæo E. Hintz $(1, \mathrm{MNHN})$.

Remarks: Only two of the seven specimens mentioned by Hintz (1908) have been located. Despite being an unavailable name, lectotypes must be designated for the taxon Phlogistus blackburni Hintz (the same lectotypes automatically represent its replacement name Phlogistus bicolor BARTLETT).

Phlogistus elongatus HINTZ, 1908

(Fig. 2)

Phlogistus elongatus Hintz, 1908: 713. Type locality: New South Wales.

Holotype (fixed by monotypy): N S Wales, Stauding. [Staudinger] / Coll. S. Schenkling / elongatus Determ. E. Hintz. / Hintz det. / HOLOTYPUS / DEI Col-01974 (SDEI).

Remarks: Hintz (1908) mentions only one specimen, therefore the single type specimen in SDEI is the holotype, fixed by monotypy.

\section{Phlogistus kolbei HINTZ, 1908}

Phlogistus kolbei Hintz, 1908: 714. Type locality: Cape York, Queensland.

Holotype (fixed by monotypy): 44242 / Type / Cap York Daemel / kolbei Hintz Determ. E. Hintz (ZMHB).

Remarks: HinTz (1908) mentions only one specimen, therefore the single type specimen in SDEI is the holotype, fixed by monotypy.

\section{Phlogistus niger HINTZ, 1908}

(Fig. 3)

Phlogistus niger Hintz, 1908: 712. Type locality: Kuranda, Queensland.

Lectotype (here designated): Kuranda F. J. Dodd / niger Determ. E. Hintz. / Hintz det. / DEI Col-01967 (SDEI). Paralectotypes (here designated): Kuranda Qld F. J. Dodd / Type / niger Hintz Determ. E. Hintz / Museum Paris 1952 Coll R. Oberthur (1, MNHN); Kuranda Qld (1, MNHN); N. Queensland (4, MNHN); Kuranda F. J. Dodd / Hintz det. / DEI Col-01968 (1, SDEI); N. Holl / Coll. Kraatz / Hintz det. / DEI Col-01969 (1, SDEI).

Remarks: All nine of the specimens mentioned by HINTZ (1908) have been located.

Phlogistus punctatus HINTZ, 1908

(Fig. 4)

Phlogistus punctatus Hintz, 1908: 713. Type locality: Kuranda, Queensland. 
Lectotype (here designated): Kuranda Qld F. J. Dodd / punctatus Determ. E. Hintz / Hintz det. / SYNTYPUS / DEI Col-01975 (SDEI).

Paralectotypes (here designated): Kuranda Qld F. J. Dodd / Type / punctatus Determ. E. Hintz / ex Musæo E. Hintz (1, MNHN); Kuranda Qld F. J. Dodd / Type / ex Musæo E. Hintz / Museum Paris 1952 Coll R. Oberthur (2, MNHN); Kuranda Qld F. J. Dodd / Hintz det. / SYNTYPUS / DEI Col-01976 (1, SDEI); Kuranda Qld F. J. Dodd / Hintz det. / SYNTYPUS / DEI Col-01977 (1, SDEI); Kuranda Qld F. J. Dodd / Hintz det. / SYNTYPUS / DEI Col-01978 (1, SDEI); Kuranda Qld F. J. Dodd / Hintz det. / SYNTYPUS / DEI Col-01979 (1, SDEI).

Remarks: Only eight of the 17 specimens mentioned by HinTZ (1908) have been located.

\section{Phlogistus rotundopunctatus HINTZ, 1908}

(Fig. 5)

Phlogistus rotundopunctatus Hintz, 1908: 714. Type locality: Kuranda, Queensland.

Lectotype (here designated): Kuranda Qld F. J. Dodd / Type / rotundopunctatus Determ. E. Hintz / ex Musæo E. Hintz / TYPE (MNHN).

Paralectotype (here designated): Kuranda Qld F. J. Dodd / rotundopunctatus Determ. E. Hintz / Hintz det. / SYNTYPUS / DEI Col-01988 (1, SDEI).

Remarks. Two of the three specimens mentioned by HinTZ (1908) have been located.

\section{Phlogistus schenklingi HINTZ, 1908}

(Fig. 6)

Phlogistus schenklingi Hintz, 1908: 715. Type locality: New South Wales.

Lectotype (here designated): Australien Heyne Brothers / schenklingi Determ. E. Hintz. / Hintz det. / DEI Col-01989 / SYNTYPUS (SDEI).

Paralectotypes (here designated): N. Holl. / Coll. Kraatz / Hintz det. / DEI Col-01990 / SYNTYPUS (1, SDEI); Austral. / Coll. Kraatz / Hintz det. / DEI Col-01991 / SYNTYPUS (1, SDEI); Aust. / Coll. Hacker / Hintz det. / DEI Col-01992 / SYNTYPUS (1, SDEI); 41195 / N. H. [? - illegible] / Type / schenklingi Determ. E. Hintz. (1, ZMHB).

Remarks: Only five of the 13 specimens mentioned by Hintz (1908) have been located.
Phlogistus schulzi HINTZ, 1908

(Fig. 7)

Phlogistus schulzi HıNTz, 1908: 712. Type locality: Australia.

Lectotype (here designated): N. Holl. / Coll. Kraatz / schulzi Determ. E. Hintz. / DEI Col-01963 / SYNTYPUS (SDEI).

Paralectotypes (here designated): N. Holl. / Type / schulzi Determ. E. Hintz / ex Musæo E. Hintz (1, MNHN); 44243 / Type Phlogistus schulzi Determ. E. Hintz. / Cap York (1, ZMHB); Kuranda Qld F. J. Dodd / Hintz det. / DEI Col-01964 / SYNTYPUS (1, SDEI); Kuranda Qld F. J. Dodd / Hintz det. / DEI Col-01966 / SYNTYPUS (1, SDEI); Austral. / Coll. Kraatz / Hintz det. / DEI Col-01965 / SYNTYPUS (1, SDEI).

Remarks: Six of the seven specimens mentioned by Hintz (1908) have been located.

Phlogistus speciosus HINTZ, 1908

(Fig. 8)

Phlogistus speciosus Hintz, 1908: 713. Type locality: Kuranda, Queensland.

Lectotype (here designated): Kuranda Qld F. J. Dodd / speciosus Determ. E. Hintz. / Hintz det. / DEI Col-01970 / SYNTYPUS (SDEI).

Paralectotypes (here designated): Kuranda Qld F. J. Dodd / Hintz det. / DEI Col-01971 / SYNTYPUS (1, SDEI); Kuranda Qld F. J. Dodd / Hintz det. / DEI Col-01972 / SYNTYPUS (1, SDEI); Kuranda Qld F. J. Dodd / Hintz det. / DEI Col-01973 / SYNTYPUS (1, SDEI); N. Queensland E. Weiske / Type / speciosus Determ. E. Hintz. / Museum Paris 1952 Coll R. Oberthur (1, MNHN); Kuranda Qld F. J. Dodd / Type / ex Musæo E. Hintz / Museum Paris 1952 Coll R. Oberthur (1, MNHN); Endeavour River Queensland / Type / ex Musæo E. Hintz / Museum Paris 1952 Coll R. Oberthur (1, MNHN).

Remarks: Seven of the 11 specimens mentioned by HINTZ (1908) have been located.

\section{Acknowledgements}

For facilitating visits to collections in their care, and authorizing loans of specimens, I thank Antoine Mantilleri (MNHN), Stephan Blank (SDEI) and Manfred Uhlig (ZMHB). I thank Christine Lambkin (Queensland Museum, Brisbane) for financing the study trip to Europe in 2011 with funds from the ABRS Bush Blitz Tactical Taxonomy grant TTC210-04. 


\section{References}

BARTLETT, J. S. 2009: A replacement name for Phlogistus blackburni Hintz, 1908 (Coleoptera: Cleridae). Australian Entomologist 36 (3): 97-98.

Groll, E. K. 2013: Biografien der Entomologen der Welt: Online-Databank-Version 6.0-Senckenberg Deutsches Entomologisches Institut.

Hintz, E. 1902a: Neue Cleriden aus Deutsch-Ostafrika. Deutsche Entomologische Zeitschrift $1902(1)$ 177-192.

Hintz, E. 1902b: Beitrag zur Kenntniss der palaarktischen Cleriden. - Deutsche Entomologische Zeitschrift 1902 (1): 227-230.

Hintz, E. 1905: Neue Cleriden aus Westfrika. - Deutsche Entomologische Zeitschrift 1905 (2): 305-314.

Hintz, E. 1907: Neue Cleriden aus Deutsch-Ostafrika. Deutsche Entomologische Zeitschrift 1907 (1): 25-28.

Hintz, E. 1908: Das Cleridengenus Phlogistus GorH. (Col.). - Deutsche Entomologische Zeitschrift 1908 (6): 708-715.
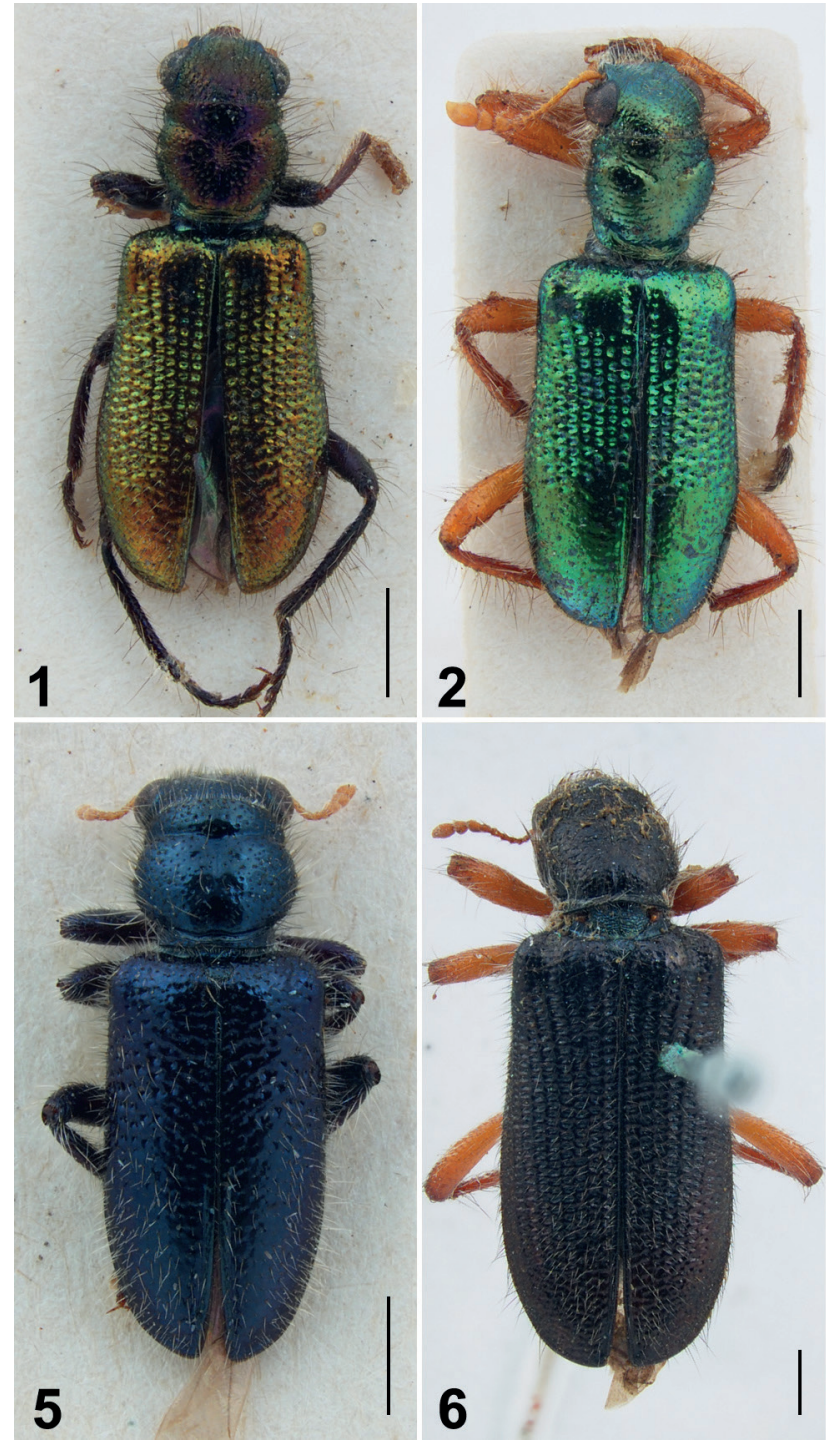

Hintz, E. 1911: Die Cerambycidengattung Sternotomis Perch. (Col.). - Deutsche Entomologische Zeitschrift 1911: 573-583.

Hintz, E. 1912: Das Cerambycidengenus Chariesthes Chevr. (Col.). - Deutsche Entomologische Zeitschrift 1912: 643-648.

Hintz, E. 1913: Neue afrikanische Cerambyciden (Col.). Deutsche Entomologische Zeitschrift 1913: 195-205.

Hintz, E. 1919: Lief. 13. Cerambyciden. En: Ergebnisse der Zweiten Deutschen Zentral-Afrika-Expedition 1910-1911 unter Führung Adolf Friedrichs, Herzogs zu Mecklenburg. Bd. 1. - Zoologie, Leipzig.

International Commission For Zoological Nomenclature (ICZN) 1999: International Code of Zoological Nomenclature. Fourth Edition. - International Trust for Zoological Nomenclature, London.

STRAND, E. 1917: Ueber einige von Herrn E. Hintz gesammelte äthiopische Hymenoptera und Lepidoptera. - Entomologische Mitteilungen 6: 34-43.
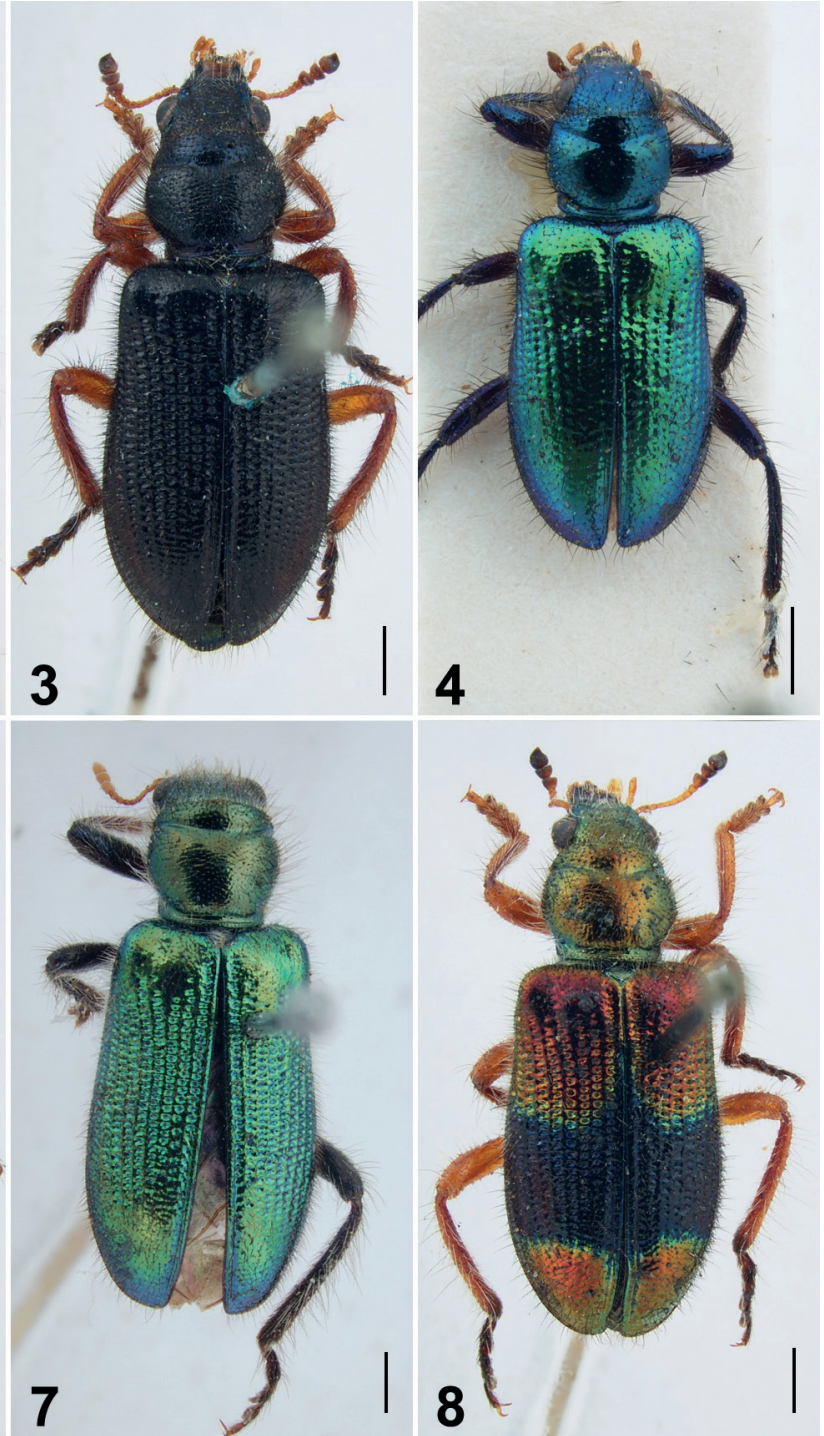

Figs 1-8: (1) Phlogistus blackburni Hintz Lectotype; (2) Phlogistus elongatus Hintz Holotype; (3) Phlogistus niger Hintz Lectotype; (4) Phlogistus punctatus Hintz Lectotype; (5) Phlogistus rotundopunctatus Hintz Paralectotype; (6) Phlogistus schenklingi Hintz Lectotype; (7) Phlogistus schulzi Hintz Lectotype; (8) Phlogistus speciosus Hintz Lectotype [scale bars = $1 \mathrm{~mm}$ ]. 